\title{
Assessment of $\mathrm{H} 3 \mathrm{~K} 27 \mathrm{me} 3$ immunohistochemistry and combination of NF1 and p16 deletions by fluorescence in situ hybridization in the differential diagnosis of malignant peripheral nerve sheath tumor and its histological mimics
}

Shintaro Sugita ${ }^{1}$, Tomoyuki Aoyama ${ }^{1}$, Makoto Emori ${ }^{2}$, Tomomi Kido ${ }^{1}$, Tomoko Takenami ${ }^{1}$, Kodai Sakuraba $^{1}$, Kotomi Terai ${ }^{1}$, Taro Sugawara ${ }^{1}$, Mitsuhiro Tsujiwaki ${ }^{1}$ and Tadashi Hasegawa ${ }^{1 *}$

\begin{abstract}
Background: A definitive diagnosis of malignant peripheral nerve sheath tumor (MPNST) is challenging, especially in cases without neurofibromatosis 1 (NF1), because MPNST lacks specific markers on immunohistochemistry (IHC).

Methods: We performed IHC for histone 3 trimethylated on lysine 27 (H3K27me3) and evaluated the percentage of cells with H3K27me3 loss using measured values at 10\% intervals, categorized as complete loss (100\% of tumor cells lost staining), partial loss (10\% to $90 \%$ of tumor cells lost staining), and intact (no tumor cells lost staining). We conducted fluorescence in situ hybridization (FISH) for NF1 and p16 deletions comparing 55 MPNSTs and 35 nonMPNSTs, consisting of 9 synovial sarcomas (SSs), 8 leiomyosarcomas (LMSs), 10 myxofibrosarcomas (MFSs), and 8 undifferentiated pleomorphic sarcomas (UPSs). We assessed the percentage of cells with homozygous and heterozygous deletions and defined "deletion" if the percentage of either the NF1 or p16 deletion signals was greater than $50 \%$ of tumor cells.
\end{abstract}

Results: Among the 55 MPNSTs, 23 (42\%) showed complete H3K27me3 loss and 32 (58\%) exhibited partial loss or intact. One each of the 9 SSs (11\%), 8 LMSs (12\%), and 8 UPSs (12\%) showed complete H3K27me3 loss and many non-MPNSTs exhibited intact or partial H3K27me3 loss. Among the 55 MPNSTs, 33 (60\%) and 44 (80\%) showed NF1 or p16 deletion, respectively. Co-deletion of NF1 and p16 was observed in 29 (53\%) MPNSTs. Among the 23 MPNTSs showing H3K27me3 complete loss, 18 (78\%) and 20 (87\%) exhibited NF1 or p16 deletion, respectively. Among the 32 MPNSTs with H3K27me3 partial loss or intact, 15 (47\%) and 24 (75\%) exhibited NF1 or p16 deletion, respectively. The frequency of NF1 and/or p16 deletion tended to be lower in non-MPNSTs than in MPNSTs. Approximately $90 \%$ of MPNSTs included cases with H3K27me3 complete loss and cases showing H3K27me3 partial loss or intact with

\footnotetext{
*Correspondence: hasetada@sapmed.ac.jp

${ }^{1}$ Department of Surgical Pathology, Sapporo Medical University, School of Medicine, South 1, West 16, Chuo-ku, Sapporo, Hokkaido 060-8543, Japan

Full list of author information is available at the end of the article
}

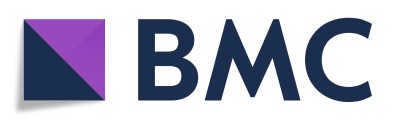

(- The Author(s). 2021 Open Access This article is licensed under a Creative Commons Attribution 4.0 International License, which permits use, sharing, adaptation, distribution and reproduction in any medium or format, as long as you give appropriate credit to the original author(s) and the source, provide a link to the Creative Commons licence, and indicate if changes were made. The images or other third party material in this article are included in the article's Creative Commons licence, unless indicated otherwise in a credit line to the material. If material is not included in the article's Creative Commons licence and your intended use is not permitted by statutory regulation or exceeds the permitted use, you will need to obtain permission directly from the copyright holder. To view a copy of this licence, visit http://creativecommons.org/licenses/by/4.0/ The Creative Commons Public Domain Dedication waiver (http://creativecommons.org/publicdomain/zero/1.0/) applies to the data made available in this article, unless otherwise stated in a credit line to the data. 
NF1 and/or p16 deletion. Approximately 50\% of MPNSTs showed co-deletion of NF1 and p16 regardless of H3K27me3 loss.

Conclusions: FISH for NF1 and p16 deletions, frequently observed in high-grade MPNSTs, might be a useful ancillary diagnostic tool for differentiating MPNST from other mimicking spindle cell and pleomorphic sarcomas.

Keywords: Malignant peripheral nerve sheath tumor, NF1 deletion, p16 deletion, Fluorescence in situ hybridization, H3K27me3, Immunohistochemistry

\section{Background}

Malignant peripheral nerve sheath tumor (MPNST) is characterized by differentiation toward peripheral nerve sheath tissue. Approximately 50\% of MPNST cases are associated with neurofibromatosis 1 (NF1), which is the most important clinical parameter for a definitive diagnosis of MPNST. Diagnosis of MPNST without NF1 is sometimes challenging because there are currently no established markers for MPNST on immunohistochemistry (IHC). Classically, MPNSTs express S-100 protein sparsely, but clearly, on IHC. However, the intensity of S-100 protein expression is sometimes markedly diminished and non-specific S-100 protein expression is often observed in other sarcomas. Recent studies have revealed that histone 3 trimethylated on lysine 27 (H3K27me3) is a useful diagnostic marker for MPNST [1, 2]. MPNST often shows complete or so-called "mosaic" loss of H3K27me3 expression on IHC, and loss of H3K27me3 is the basis for diagnosing MPNST. However, approximately one-third of cases retain H3K27me3 expression, and the evaluation of $\mathrm{H} 3 \mathrm{~K} 27 \mathrm{me} 3$, especially the detailed ratio of mosaic loss, is empirically difficult and seems to be somewhat non-objective. In the clinical setting, we make a final diagnosis of MPNST by the combination of H3K27me3 expression and classic diagnostic hallmarks including S-100 protein expression and association with NF1.

It has been reported that $N F 1$ deletion is characteristic of MPNST. NF1 deletion can be detected by fluorescence in situ hybridization (FISH) and is useful in pathological diagnosis using formalin-fixed and paraffinembedded specimens. Perry et al. found that NF1 was characteristically deleted in MPNST cases in a study of benign peripheral nerve sheath tumors and MPNSTs [3]. In addition, Suzuki et al. showed that NF1 deletion FISH was useful for diagnosis in diagnostically challenging cases of intraosseous MPNST without NF1 [4].

Perry et al. first revealed the diagnostic utility of a FISH assay for NF1 and $p 16$ in MPNST [5]. They performed a dual-color FISH assay with 22 MPNSTs and benign and malignant non-peripheral nerve sheath tumors (non-MPNSTs) including 13 plexiform schwannomas, 5 cellular schwannomas, 8 synovial sarcomas, 6 fibrosarcomas, and 13 hemangiopericytomas. They demonstrated the specific deletion of NF1 and $p 16$ in MPNST and indicated that homozygous and heterozygous deletion of $p 16$ might distinguish MPNST from benign nerve sheath tumor and other histologically mimicking sarcomas. From the genetic aspect, p16/ $C D K N 2 A$ inactivation is considered a key event in the occurrence and progression of MPNST. Loss of the $p 16 /$ $C D K N 2 A$ locus at $9 \mathrm{p} 21$ is one of the earliest events in the malignant transformation of neurofibromas. The expression of p16 on IHC is sometimes decreased in MPNST [6]. Atypical and conventional neurofibromas also exhibit the loss of nuclear p16 expression. Therefore, $p 16 / C D K N 2 A$ inactivation may be an early change in malignant progression [7].

In this study, aiming for more accurate differential diagnosis, we examined the diagnostic utility of FISH for NF1 and p16 deletions in the differential diagnosis of MPNST and its mimicking spindle cell and pleomorphic sarcomas, especially in mosaic loss cases in which the interpretation of H3K27me3 can be difficult.

\section{Methods}

\section{Sample selection}

This study was performed with the approval of the Institutional Review Board (IRB) of Sapporo Medical University Hospital (No. 272-107). The IRB approved an optout informed consent approach for a retrospective, noninterventional study. We selected 55 MPNST cases from our pathological archives. Twenty cases were NF1associated MPNSTs (NF1Ms) and 35 cases were nonNF1-associated MPNSTs (NNF1Ms). We determined histological grade using the parameters of tumor differentiation, mitotic figures, and tumor necrosis according to the Fédération Nationale des Centres de Lutte Contre le Cancer grading system. We performed hematoxylin and eosin staining using 3 - $\mu \mathrm{m}$-thick sections. We reviewed all hematoxylin and eosin-stained slides and previously stained IHC slides in individual cases. Next, we selected 35 non-MPNST sarcomas that needed to be distinguished from MPNST, comprising 9 synovial sarcomas (SSs), 8 leiomyosarcomas (LMSs), 10 myxofibrosarcomas (MFSs), and 8 undifferentiated pleomorphic sarcomas (UPSs). The histopathological criteria for nonMPNSTs were as follows. SS showed fascicular 
proliferation of uniform spindle cells. The tumor cells were positive for cytokeratin and/or epithelial membrane antigen to varying degrees on IHC. All SS cases had been shown to have SS18 rearrangement by FISH. LMS exhibited interlacing fascicules of spindle cells with cigar-shaped nuclei and eosinophilic cytoplasm. The tumor cells expressed at least two of three myogenic markers $(\alpha$-smooth muscle actin, desmin, and musclespecific actin) on IHC. MFS was characterized by multinodular growth of hypocellular proliferating atypical spindle cells with prominent elongated, curvilinear, thinwalled blood vessels in a myxoid background. UPS exhibited a pattern-less growth of severely atypical spindle or pleomorphic cells without any differentiation toward specific tissue on IHC.

We checked the immunoreactivity of previously performed IHC for S-100 protein in all 55 MPNST cases. As a result, 50 cases (approximately 90\%) expressed S100 protein on IHC. Twenty-eight cases demonstrated sparse or focal expression of S-100 protein. On the other hand, 22 cases were diffusely positive for S-100 protein.

\section{H3K27me3 IHC}

We performed IHC for H3K27me3 using representative sections from formalin-fixed paraffin-embedded tissues from MPNST and non-MPNST cases. These tissues were sliced into 3 - $\mu \mathrm{m}$-thick sections and examined with an automated IHC system at Sapporo Medical University Hospital. All slides were loaded into a PT Link Module (Agilent Technologies, Santa Clara, CA) and subjected to a heat-induced antigen-retrieval protocol with EnVision FLEX Target Retrieval Solution (Agilent Technologies) before being transferred to an Autostainer Link 48 (Agilent Technologies). We used antibodies against H3K27me3 (C36B11, 1:200 dilution; Cell Signaling Technology, Danvers, MA). We determined H3K27me3 loss when we recognized the loss of H3K27me3 nuclear staining in the tumor cells. We evaluated the percentage of cells with $\mathrm{H} 3 \mathrm{~K} 27 \mathrm{me} 3$ loss using measured values at $10 \%$ intervals and categorized it as complete loss $(100 \%$ of tumor cells lost staining), partial loss ( $10 \%$ to $90 \%$ of tumor cells lost staining), and intact (no tumor cells lost staining).

\section{FISH}

We performed FISH using the commercially available probes MD NF1 (17q11)/MPO (17q22) (Leica, Wetzlar, Germany) for NF1 deletion and Vysis LSI CDKN2A SpectrumOrange/CEP9 SpectrumGreen Probes (Abbott, Abbott Park, IL) for $p 16$ (CDKN2A) deletion. Each of the probes was labeled with red or green dye for the target or control locus, respectively. FISH was performed as described previously [8]. In brief, the specimens were tumor tissues in $4-\mu \mathrm{m}$-thick slices on glass slides. We first selected an area showing representative histology and marked a 5-mm circle with a marker pen on the glass slide. We used a PathVysion HER-2 DNA Probe Kit (Abbott) and followed the manufacturer's procedure, with modifications: baking $\left(60^{\circ} \mathrm{C}\right.$ for $\left.1 \mathrm{~h}\right)$, deparaffinization, target gene activation $(20$ min with $0.2 \mathrm{M} \mathrm{HCl}$ followed by $80^{\circ} \mathrm{C}$ for $30 \mathrm{~min}$ with pretreatment solution), enzyme treatment $\left(37^{\circ} \mathrm{C}\right.$ for $60 \mathrm{~min}$ with protease solution), re-fixation (10 min with $10 \%$ formalin neutral buffer solution), denaturation $\left(72^{\circ} \mathrm{C}\right.$ for $5 \mathrm{~min}$ with denaturation solution), washing and dehydration (1 min each in $70 \%, 85 \%$, and $100 \%$ ethanol), hybridization with $10 \mathrm{~mL}$ DNA probe solution $\left(90^{\circ} \mathrm{C}\right.$ for $5 \mathrm{~min}$, followed by $37^{\circ} \mathrm{C}$ for $48 \mathrm{~h}$ ), and washing with post-hybridization wash buffer $\left(72^{\circ} \mathrm{C}\right.$ for $\left.2 \mathrm{~min}\right)$. For counterstaining, $10 \mu \mathrm{L}$ of 4,6-diamidino-2-phenylindole was added. The slides were cover-slipped for viewing under a fluorescence microscope. We counted 50 tumor nuclei and calculated the percentage of cells with deletion signals. Deletion signals were categorized as homozygous deletion, heterozygous deletion, and monosomy. Homozygous deletion was defined as complete deletion of both alleles of the target locus and cells showed only control green signals. Heterozygous deletion was defined as alternate deletion of one of the alleles of the target locus, and so the number of red signals for the target locus was less than the number of green signals for the control locus. Tumor cells with monosomy had one allele, which showed a pair of red and green signals. Monosomy was considered a variation of heterozygous deletion. We defined "deletion" if the percentage of either the NF1 or $p 16$ deletion signals was greater than $50 \%$ of tumor cells.

\section{Statistical analysis}

To examine whether there was a difference in the IHC and FISH results between low-grade and high-grade MPNST, we performed statistical analysis by Fisher's exact test using IBM SPSS Statistics 25 software (IBM SPSS Statistics, Chicago, IL). For all analysis, differences at $\mathrm{P}<0.05$ were considered statistically significant.

\section{Results}

\section{Histological examinations}

Histologically, MPNST consisted mainly of fascicular and storiform proliferation of spindle cells that had enlarged oval to spindle nuclei with moderate to severe nuclear atypia and nuclear pleomorphism (Fig. 1a, d). The tumor showed a marble pattern intermingled with solid and edematous to myxoid areas. Mitotic figures were frequently observed with necrotic foci. Some MPNSTs consisted of the solid proliferation of round cells. Some cases also showed a myxoid morphology, and glandular differentiation foci and scattered rhabdomyoblasts (malignant Triton tumor) were observed. Our cohort 


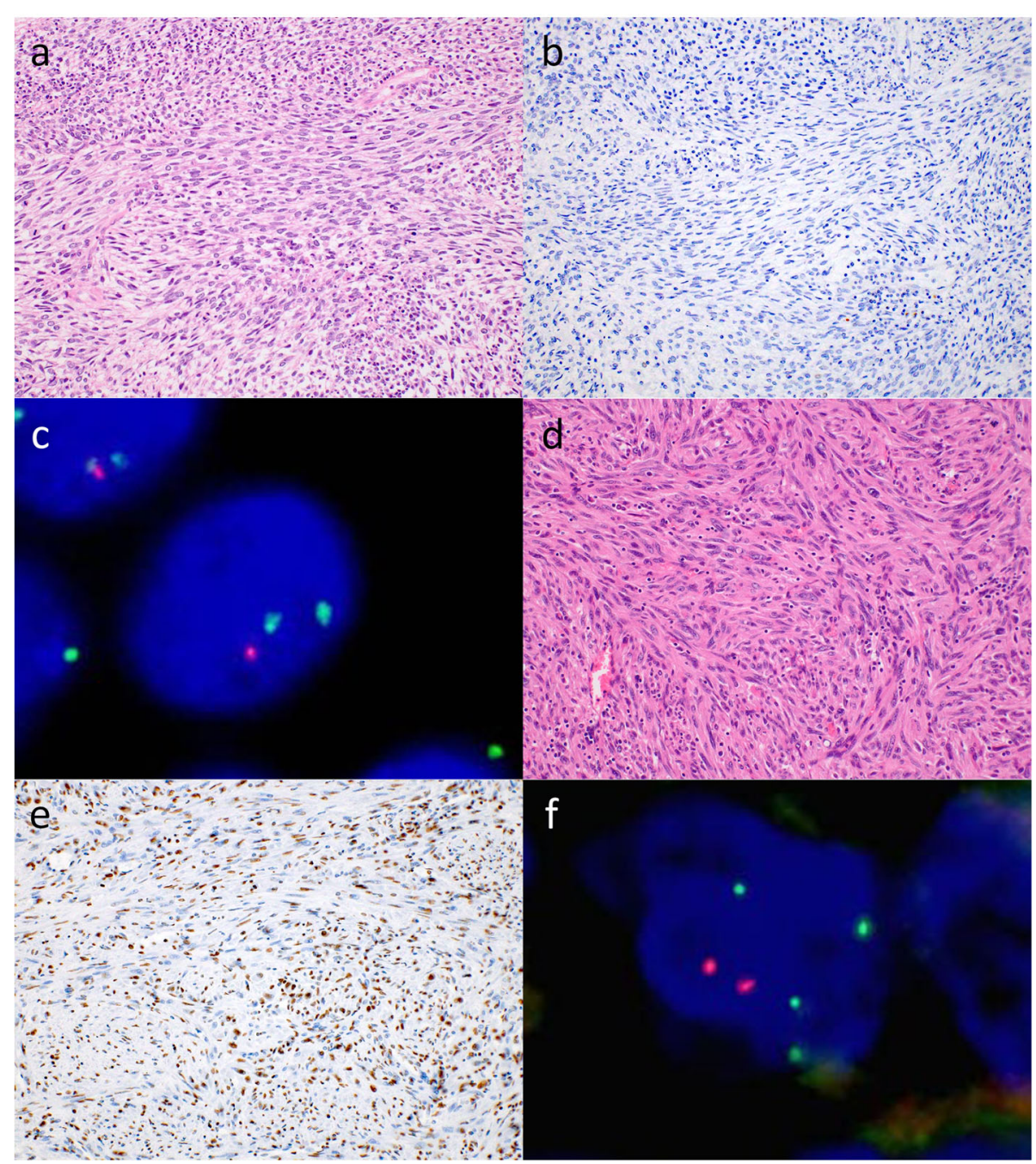

Fig. 1 Pathological findings including morphology, immunohistochemistry (IHC), and fluorescence in situ hybridization (FISH) of malignant peripheral nerve sheath tumor (MPNST) cases. a. MPNST with fascicular proliferation of spindle cells that had enlarged oval to spindle nuclei with moderate to severe nuclear atypia (NF1M-20). b. IHC for histone 3 trimethylated on lysine 27 (H3K27me3). The tumor cells were completely negative for H3K27me3 on IHC (NF1M-20). c. FISH for p16 deletion. The tumor nuclei showed heterozygous deletion of p16. Two green signals indicating a control locus and one red signal indicating a target locus were found (NF1M-20). d. Another case of MPNST also exhibited fascicular and storiform proliferation of spindle cells with enlarged spindle nuclei with moderate to severe nuclear atypia (NF1M-6). e. IHC for H3K27me3. A majority of tumor cells were positive for H3K27me3 on IHC, although H3K27me3-negative tumor cells were focally intermingled. The tumor showed partial loss of H3K27me3 (NF1M-6). f. FISH for neurofibromatosis 1 (NF1) deletion. Tumor nuclei showing heterozygous deletion of NF1. The number of red signals (two) for the target locus was less than the number of green signals (four) for the control locus (NF1M-6)

consisted of 7 cases of low-grade/grade 1 MPNST and 48 cases of high-grade/grades 2 and 3 MPNST.

\section{H3K27me3 IHC}

We performed H3K27me3 IHC in 55 cases of MPNST (20 NF1Ms and 35 NNF1Ms) and 35 non-MPNST cases (Table 1). Among the 55 MPNSTs, 23 (42\%) showed complete loss of H3K27me3 on IHC (Figs. 1b, 2a) and 32 (58\%) exhibited partial loss or intact (Figs. 1e, 2a). Among the 20 NF1Ms and 35 NNF1Ms, 11 (55\%) (Fig. 2b) and 12 (34\%) (Fig. 2c) showed complete loss of H3K27me3, respectively. There was no difference in the staining pattern between the conventional MPNST cases and those with heterologous components (malignant Triton tumor). On the other hand, one each of the 9 SSs (11\%), 8 LMSs (12\%), and 8 UPSs (12\%) showed complete loss of H3K27me3, and many non-MPNSTs exhibited intact or partial loss of H3K27me3 (Fig. 3).

\section{FISH}

The results for NF1 and $p 16$ deletion FISH of all cases are described in Table 1. Among the 55 MPNSTs, 33 $(60 \%)$ and $44(80 \%)$ showed NF1 or p16 deletion, respectively. Co-deletion of NF1 and p16 was observed in 
Table 1 Status of H3K27me3 loss, NF1 deletion, and p16 deletion in individual cases of MPNST and non-MPNST

\begin{tabular}{|c|c|c|c|c|c|c|c|c|c|c|c|}
\hline Case & $\begin{array}{l}\text { H3K27me3 } \\
\text { loss (\%) }\end{array}$ & $\begin{array}{l}\text { NF1 del } \\
(\%)\end{array}$ & $\begin{array}{l}\text { p16 del } \\
(\%)\end{array}$ & Case & $\begin{array}{l}\text { H3K27me3 loss } \\
(\%)\end{array}$ & $\begin{array}{l}\text { NF1 del } \\
(\%)\end{array}$ & $\begin{array}{l}\text { p16 del } \\
(\%)\end{array}$ & Case & $\begin{array}{l}\text { H3K27me3 loss } \\
(\%)\end{array}$ & $\begin{array}{l}\text { NF1 del } \\
(\%)\end{array}$ & $\begin{array}{l}\text { p16 del } \\
(\%)\end{array}$ \\
\hline $\begin{array}{l}\text { NNF1M- } \\
1\end{array}$ & 0 & 0 & 0 & $\begin{array}{l}\text { NF1M- } \\
1\end{array}$ & 0 & 6 & 48 & SS-1 & 10 & 24 & 28 \\
\hline $\begin{array}{l}\text { NNF1M- } \\
2\end{array}$ & 0 & 20 & 32 & $\begin{array}{l}\text { NF1M- } \\
2\end{array}$ & 0 & 48 & 94 & SS-2 & 10 & 32 & 20 \\
\hline $\begin{array}{l}\text { NNF1M- } \\
3\end{array}$ & 0 & 47 & 100 & $\begin{array}{l}\text { NF1M- } \\
3\end{array}$ & 10 & 12 & 40 & SS-3 & 10 & 70 & 40 \\
\hline $\begin{array}{l}\text { NNF1M- } \\
4\end{array}$ & 0 & 20 & 58 & $\begin{array}{l}\text { NF1M- } \\
4\end{array}$ & 10 & 96 & 96 & SS-4 & 40 & 8 & 20 \\
\hline $\begin{array}{l}\text { NNF1M- } \\
5\end{array}$ & 0 & 20 & 56 & $\begin{array}{l}\text { NF1M- } \\
5\end{array}$ & 10 & 84 & 88 & SS-5 & 50 & 18 & 16 \\
\hline $\begin{array}{l}\text { NNF1M- } \\
6\end{array}$ & 0 & 38 & 76 & $\begin{array}{l}\text { NF1M- } \\
6\end{array}$ & 10 & 72 & 98 & SS-6 & 60 & 6 & 32 \\
\hline $\begin{array}{l}\text { NNF1M- } \\
7\end{array}$ & 0 & 38 & 100 & $\begin{array}{l}\text { NF1M- } \\
7\end{array}$ & 20 & 6 & 68 & SS-7 & 70 & 10 & 16 \\
\hline $\begin{array}{l}\text { NNF1M- } \\
8\end{array}$ & 0 & 88 & 56 & $\begin{array}{l}\text { NF1M- } \\
8\end{array}$ & 70 & 90 & 30 & SS-8 & 70 & 22 & 14 \\
\hline $\begin{array}{l}\text { NNF1M- } \\
9\end{array}$ & 10 & 0 & 76 & $\begin{array}{l}\text { NF1M- } \\
9\end{array}$ & 90 & 98 & 14 & SS-9 & 100 & 50 & 42 \\
\hline $\begin{array}{l}\text { NNF1M- } \\
10\end{array}$ & 10 & 12 & 14 & $\begin{array}{l}\text { NF1M- } \\
10\end{array}$ & 100 & 38 & 66 & $\begin{array}{l}\text { LMS- } \\
1\end{array}$ & 10 & 84 & 96 \\
\hline $\begin{array}{l}\text { NNF1M- } \\
11\end{array}$ & 10 & 10 & 70 & $\begin{array}{l}\text { NF1M- } \\
11\end{array}$ & 100 & 20 & 56 & $\begin{array}{l}\text { LMS- } \\
2\end{array}$ & 20 & 12 & 10 \\
\hline $\begin{array}{l}\text { NNF1M- } \\
12\end{array}$ & 10 & 12 & 90 & $\begin{array}{l}\text { NF1M- } \\
12\end{array}$ & 100 & 96 & 14 & $\begin{array}{l}\text { LMS- } \\
3\end{array}$ & 20 & 38 & 98 \\
\hline $\begin{array}{l}\text { NNF1M- } \\
13\end{array}$ & 10 & 24 & 74 & $\begin{array}{l}\text { NF1M- } \\
13\end{array}$ & 100 & 84 & 84 & $\begin{array}{l}\text { LMS- } \\
4\end{array}$ & 30 & 26 & 28 \\
\hline $\begin{array}{l}\text { NNF1M- } \\
14\end{array}$ & 10 & 68 & 66 & $\begin{array}{l}\text { NF1M- } \\
14\end{array}$ & 100 & 60 & 94 & $\begin{array}{l}\text { LMS- } \\
5\end{array}$ & 40 & 14 & 90 \\
\hline $\begin{array}{l}\text { NNF1M- } \\
15\end{array}$ & 10 & 80 & 82 & $\begin{array}{l}\text { NF1M- } \\
15\end{array}$ & 100 & 62 & 62 & $\begin{array}{l}\text { LMS- } \\
6\end{array}$ & 70 & 18 & 18 \\
\hline $\begin{array}{l}\text { NNF1M- } \\
16\end{array}$ & 10 & 60 & 72 & $\begin{array}{l}\text { NF1M- } \\
16\end{array}$ & 100 & 60 & 80 & $\begin{array}{l}\text { LMS- } \\
7\end{array}$ & 90 & 14 & 36 \\
\hline $\begin{array}{l}\text { NNF1M- } \\
17\end{array}$ & 10 & 88 & 78 & $\begin{array}{l}\text { NF1M- } \\
17\end{array}$ & 100 & 76 & 88 & $\begin{array}{l}\text { LMS- } \\
8\end{array}$ & 100 & 12 & 22 \\
\hline $\begin{array}{l}\text { NNF1M- } \\
18\end{array}$ & 10 & 62 & 88 & $\begin{array}{l}\text { NF1M- } \\
18\end{array}$ & 100 & 96 & 98 & $\begin{array}{l}\text { MFS- } \\
1\end{array}$ & 10 & 24 & 90 \\
\hline $\begin{array}{l}\text { NNF1M- } \\
19\end{array}$ & 20 & 44 & 12 & $\begin{array}{l}\text { NF1M- } \\
19\end{array}$ & 100 & 78 & 58 & $\begin{array}{l}\text { MFS- } \\
2\end{array}$ & 20 & 8 & 84 \\
\hline $\begin{array}{l}\text { NNF1M- } \\
20\end{array}$ & 30 & 100 & 64 & $\begin{array}{l}\text { NF1M- } \\
20\end{array}$ & 100 & 96 & 98 & $\begin{array}{l}\text { MFS- } \\
3\end{array}$ & 30 & 48 & 16 \\
\hline $\begin{array}{l}\text { NNF1M- } \\
21\end{array}$ & 30 & 98 & 56 & & & & & $\begin{array}{l}\text { MFS- } \\
4\end{array}$ & 30 & 4 & 96 \\
\hline $\begin{array}{l}\text { NNF1M- } \\
22\end{array}$ & 70 & 72 & 98 & & & & & $\begin{array}{l}\text { MFS- } \\
5\end{array}$ & 40 & 84 & 80 \\
\hline $\begin{array}{l}\text { NNF1M- } \\
23\end{array}$ & 80 & 56 & 68 & & & & & $\begin{array}{l}\text { MFS- } \\
6\end{array}$ & 80 & 10 & 30 \\
\hline $\begin{array}{l}\text { NNF1M- } \\
24\end{array}$ & 100 & 10 & 8 & & & & & $\begin{array}{l}\text { MFS- } \\
7\end{array}$ & 90 & 22 & 48 \\
\hline $\begin{array}{l}\text { NNF1M- } \\
25\end{array}$ & 100 & 36 & 92 & & & & & $\begin{array}{l}\text { MFS- } \\
8\end{array}$ & 90 & 46 & 80 \\
\hline $\begin{array}{l}\text { NNF1M- } \\
26\end{array}$ & 100 & 20 & 60 & & & & & $\begin{array}{l}\text { MFS- } \\
9\end{array}$ & 90 & 48 & 100 \\
\hline NNF1M- & 100 & 78 & 36 & & & & & MFS- & 90 & 54 & 98 \\
\hline
\end{tabular}


Table 1 Status of H3K27me3 loss, NF1 deletion, and p16 deletion in individual cases of MPNST and non-MPNST (Continued)

\begin{tabular}{|c|c|c|c|c|c|c|c|c|c|c|c|}
\hline Case & $\begin{array}{l}\text { H3K27me3 } \\
\text { loss (\%) }\end{array}$ & $\begin{array}{l}\text { NF1 del } \\
\text { (\%) }\end{array}$ & $\begin{array}{l}\text { p16 del } \\
(\%)\end{array}$ & Case & $\begin{array}{l}\text { H3K27me3 loss } \\
\text { (\%) }\end{array}$ & $\begin{array}{l}\text { NF1 del } \\
\text { (\%) }\end{array}$ & $\begin{array}{l}\text { p16 del } \\
(\%)\end{array}$ & Case & $\begin{array}{l}\text { H3K27me3 loss } \\
\text { (\%) }\end{array}$ & $\begin{array}{l}\text { NF1 del } \\
\text { (\%) }\end{array}$ & $\begin{array}{l}\text { p16 del } \\
\text { (\%) }\end{array}$ \\
\hline 27 & & & & & & & & 10 & & & \\
\hline $\begin{array}{l}\text { NNF1M- } \\
28\end{array}$ & 100 & 88 & 98 & & & & & UPS-1 & 0 & 30 & 40 \\
\hline $\begin{array}{l}\text { NNF1M- } \\
29\end{array}$ & 100 & 60 & 94 & & & & & UPS-2 & 0 & 66 & 96 \\
\hline $\begin{array}{l}\text { NNF1M- } \\
30\end{array}$ & 100 & 66 & 82 & & & & & UPS-3 & 10 & 46 & 44 \\
\hline $\begin{array}{l}\text { NNF1M- } \\
31\end{array}$ & 100 & 58 & 88 & & & & & UPS-4 & 10 & 40 & 14 \\
\hline $\begin{array}{l}\text { NNF1M- } \\
32\end{array}$ & 100 & 64 & 80 & & & & & UPS-5 & 10 & 76 & 74 \\
\hline $\begin{array}{l}\text { NNF1M- } \\
33\end{array}$ & 100 & 86 & 94 & & & & & UPS-6 & 10 & 52 & 94 \\
\hline $\begin{array}{l}\text { NNF1M- } \\
34\end{array}$ & 100 & 96 & 84 & & & & & UPS-7 & 80 & 6 & 52 \\
\hline $\begin{array}{l}\text { NNF1M- } \\
35\end{array}$ & 100 & 52 & 70 & & & & & UPS-8 & 100 & 40 & 44 \\
\hline
\end{tabular}

del deletion, H3K27me3 histone 3 trimethylated on lysine 27, LMS leiomyosarcoma, MFS myxofibrosarcoma, MPNST malignant peripheral nerve sheath tumor, NF1 neurofibromatosis 1, NF1M neurofibromatosis 1-associated malignant peripheral nerve sheath tumor, NNF1M non-neurofibromatosis 1-associated malignant peripheral nerve sheath tumor, SS synovial sarcoma, UPS undifferentiated pleomorphic sarcoma

29 (53\%) MPNSTs. Among the 23 MPNSTs showing H3K27me3 complete loss, 18 (78\%) and 20 (87\%) exhibited NF1 or p16 deletion, respectively. Among the 32 MPNSTs with H3K27me3 partial loss or intact, 15 (47\%) showed NF1 deletion and 24 (75\%) exhibited p16 deletion. On the other hand, although MFSs often showed p16 deletion, the frequency of NF1 and p16 deletion tended to be lower in non-MPNSTs than in MPNSTs.

On fluorescence microscopy, FISH typically detected only two green signals, indicating homozygous deletion of NF1 in MPNSTs. On the other hand, heterozygous deletion of NF1 was indicated by the lower number of red signals for the target locus compared to the number of green signals for the control locus (Fig. 1f). FISH also detected $p 16$ homozygous deletion, showing only green signals. Additionally, $p 16$ heterozygous deletion was identified, in which the number of red signals for the target locus was less than the number of green signals for the control locus (Fig. 1c).

\section{Combination of H3K27me3 IHC and NF1 and p16 deletions by FISH for differential diagnosis}

Among the 55 MPNSTs, 26 (47\%) showed H3K27me3 partial loss or intact with NF1 and/or p16 deletion. Six cases (11\%) exhibited H3K27me3 partial loss or intact without NF1 and/or $p 16$ deletion. NF1Ms and NNF1Ms also demonstrated similar trends to the results of all MPNST cases (Fig. 2). Therefore, approximately $90 \%$ of MPNSTs included cases with H3K27me3 complete loss and cases showing H3K27me3 partial loss or intact with NF1 and/or p16 deletion. Approximately 50\% of MPNS
Ts showed co-deletion of NF1 and $p 16$, regardless of the loss of H3K27me3 (Table 1 and Fig. 2). Among the nonMPNSTs, SSs included a minority of cases with H3K27me3 complete loss and cases showing H3K27me3 partial loss or intact with NF1 and/or p16 deletion. On the other hand, MFSs and UPSs mainly contained cases showing H3K27me3 partial loss or intact with/without NF1 and/or p16 deletion (Fig. 3).

\section{Statistical analysis}

There was a significant difference in the presence of NF1 and/or p16 deletion between low-grade and highgrade MPNSTs $(\mathrm{P}<0.022)$ (Table 2$)$. There was no significant difference in the presence of H3K27me3 complete loss, NF1 deletion, and p16 deletion between both groups.

\section{Discussion}

In general, a definitive diagnosis of MPNST is often difficult or challenging because the tumor fundamentally shows spindle cell morphology and does not possess any IHC markers that are completely specific for MPNST. The differential diagnosis of MPNST ranges from benign to malignant tumors [7], and so we investigated SS, LMS, MFS, and UPS. SS shows fascicular proliferation of spindle cells, especially in monophasic fibrous SS, and expresses epithelial markers (cytokeratin and epithelial membrane antigen) on IHC. Finally, SS has a specific chimeric SS18-SSX fusion gene. LMS also shows fascicular proliferation of spindle cells with cigar-like nuclei and eosinophilic cytoplasm. The tumor cells often 
a. MPNST ( $n=55)$

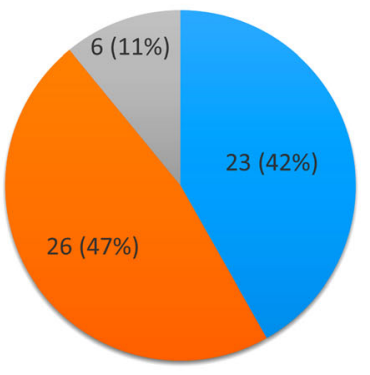

c. NNF1M ( $n=35)$

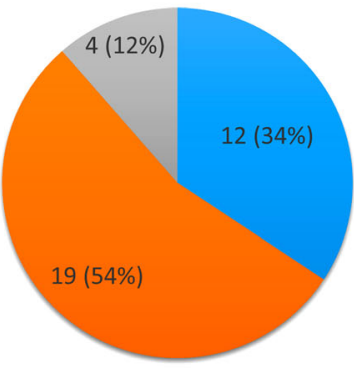

b. NF1M $(n=20)$

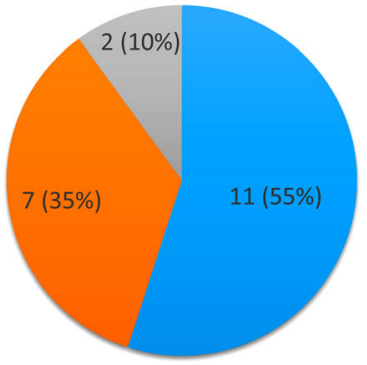

H3K27me3 complete loss

H3K27me3 partial loss or intact with NF1 and/or p16 deletion

H3K27me3 partial loss or intact without NF1 and/or $p 16$ deletion

Fig. 2 Combination of immunohistochemistry for histone 3 trimethylated on lysine 27 (H3K27me3) and fluorescence in situ hybridization for neurofibromatosis 1 (NF1) and p16 deletion in malignant peripheral nerve sheath tumor (MPNST) cases. a. Status of H3K27me3 loss and NF1 and/ or p16 deletion in all MPNST cases. A majority of MPNST cases had H3K27me3 complete loss and H3K27me3 partial loss or intact with NF1 and/or p16 deletion. b. Status of H3K27me3 loss and NF1 and/or p16 deletion in neurofibromatosis 1 (NF1)-associated MPNST (NF1M) cases. c. Status of H3K27me3 loss and NF1 and/or p16 deletion in non-NF1 associated MPNST (NNF1M) cases

express myogenic markers ( $\alpha$-smooth muscle actin, desmin, and muscle-specific actin) on IHC. MFS exhibits loose fascicular proliferation of atypical spindle cells with abundant myxoid matrix. UPS consists of markedly atypical spindle and pleomorphic cells. Neither MFS nor UPS have characteristic markers on IHC. Therefore, MPNST should always be differentiated from these tumors.

On IHC, MPNST often shows complete or mosaic loss of H3K27me3 expression. However, the specificity of the loss of H3K27me3 expression in the differential diagnosis of MPNST is not complete and one study reported that H3K27me3 cannot be used safely to differentiate between MPNST and malignant melanoma [9]. In addition, the loss of H3K27me3 expression has been confirmed in other histologic mesenchymal tumors including some extraskeletal osteosarcomas and dedifferentiated chondrosarcomas $[10,11]$.

A recent study revealed the diagnostic utility of histone H3K27 dimethylation (H3K27me2) loss in the differential diagnosis of MPNST [12]. Marchione et al. investigated H3K27me2 IHC in MPNST, K27M-mutant glioma, ependymoma, and Merkel cell carcinoma, which were characterized by the loss of H3K27me3. They demonstrated that while H3K27me3 loss is common across these tumor types, H3K27me2 loss is limited to MPNST. Moreover, they also investigated H3K27me2 IHC in cases of malignant melanoma and SS, which were histologic mimics of MPNST and showed various degrees of H3K27me3 loss. As a result, while global H3K27me3 loss was not observed in these tumors, weak and limited H3K27me3 staining was common. On the other hand, H3K27me2 staining was more clearly retained in all cases. Thus, H3K27me2 may be a useful marker for the differential diagnosis of MPNST instead of H3K27me3, the partial loss of which is particularly difficult to assess using immunoreactivity.

Indeed, the present study revealed that some nonMPNSTs showed H3K27me3 partial loss ranging from $10 \%$ to $90 \%$ of tumor cells. Among them, MPNSTs 
a. SS $(n=9)$

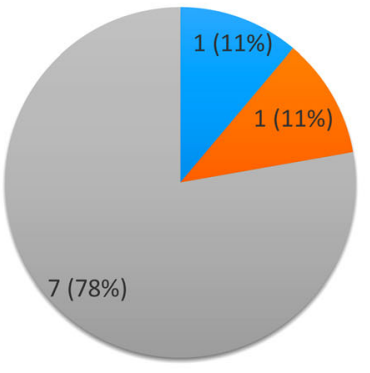

c. MFS $(n=10)$

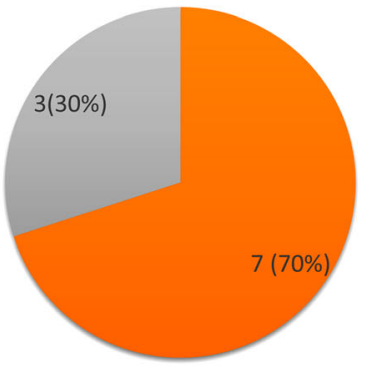

b. LMS $(n=8)$

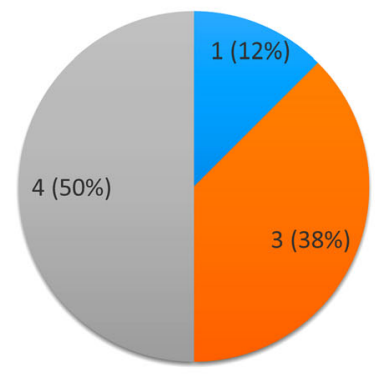

d. UPS $(n=8)$

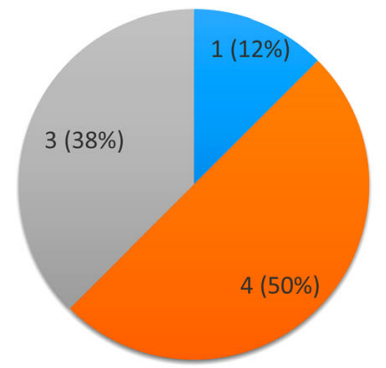

H3K27me3 complete loss

H3K27me3 partial loss or intact with NF1 and/or p16 deletion

H3K27me3 partial loss or intact without NF1 and/or $p 16$ deletion

Fig. 3 Combination of immunohistochemistry for histone 3 trimethylated on lysine 27 (H3K27me3) and fluorescence in situ hybridization for neurofibromatosis 1 (NF1) and p16 deletion in non-malignant peripheral nerve sheath tumors. a. Status of H3K27me3 loss and NF1 and/or p16 deletion in synovial sarcoma (SS) cases. A minority of SS cases showed H3K27me3 complete loss and H3K27me3 partial loss or intact with NF1 and/or p16 deletion. b. Status of H3K27me3 loss and NF1 and/or p16 deletion in leiomyosarcoma (LMS) cases. c. Status of H3K27me3 loss and NF1 and/or p16 deletion in myxofibrosarcoma (MFS) cases. A majority of MFS cases showed H3K27me3 partial loss or intact with NF1 and/or p16 deletion. d. Status of H3K27me3 loss and NF1 and/or p16 deletion in undifferentiated pleomorphic sarcoma (UPS) cases

tended to exhibit NF1 and/or $p 16$ deletion more frequently than non-MPNSTs. In particular, the combination of H3K27me3 IHC and NF1/p16 deletion FISH was able to reach an accurate diagnosis in approximately $90 \%$ of MPNST cases.

Nevertheless, there were some non-MPNST cases that were difficult to differentiate from MPNST according to the status of H3K27me3 loss and NF1 and/or p16 deletion, but these cases can be diagnosed by combining

Table 2 Status of NF1 and/or p16 deletion between low-grade and high-grade MPNSTs

\begin{tabular}{lllll}
\hline & \multicolumn{4}{l}{ NF1 and/or p16 deletion } \\
\hline Histology & Total, $n(\%)$ & Present, $n(\%)$ & Absent, $n(\%)$ & P-value \\
Low-grade MPNST & $7(12.7)$ & $4(57.1)$ & $3(42.9)$ & 0.022 \\
High-grade MPNST & $48(87.3)$ & $45(93.8)$ & $3(6.2)$ & \\
\hline
\end{tabular}

MPNST malignant peripheral nerve sheath tumor, NF1 neurofibromatosis 1 other IHC and FISH findings. For example, SS-3 and SS9 cases showed NF1 deletion, and SS-9 cases also exhibited complete loss of H3K27me3. SS is especially important for differential diagnosis from MPNST because of the monomorphic appearance of spindle cell proliferation. However, we were able to reliably distinguish these two cases from MPNST by cytokeratin expression and identifying the SS18-SSX fusion gene. In another example, some LMSs (LMS-1, 3, 5) also showed NF1 and/ or p16 deletion, although positivity for myogenic markers on IHC helped us to reach a correct diagnosis. We often find that the differential diagnosis of MFS and UPS is the most challenging because these tumors have no specific markers on IHC. A recent study revealed that somatic tumor genomic profiles by FoundationOne CDx showed a high prevalence of $p 16 / C D K N 2 A$ alterations in MPNST, MFS, and UPS [13]. These sarcomas may have some overlap of genetic features. From the clinical point of view, MFSs are relatively distinguishable 
according to their superficial localization and characteristic radiographic findings showing extension along the fascia. Ultimately, it seems that there are certain UPS cases that are tremendously difficult to distinguish from MPNST. We re-evaluated the MFS and UPS cases showing co-deletion of NF1 and $p 16$ (MFS-5, 10, UPS-2, 5, 6) and confirmed that they were morphologically typical MFS and UPS and different from MPNST when judged comprehensively. In such a situation, we need a multidisciplinary approach that combines not only pathological findings but also epidemiological, clinical, and radiographic findings for an accurate diagnosis.

It has been reported that loss of p16 expression on $\mathrm{IHC}$ is associated with poor prognosis in patients with high-grade sarcoma including MPNST $[6,14]$. In addition, $p 16$ homozygous deletion is a marker of poor prognosis in patients with Ewing sarcoma [15]. Moreover, p16 deletion has been revealed to be a biomarker for poor prognosis in patients with soft tissue sarcoma [13]. In this way, $p 16$ is an important marker of the clinical behavior of patients with soft tissue sarcoma. In this study, we revealed that the presence of NF1 and/or $p 16$ deletion was associated with the histological grade of MPNST. Although we did not investigate the association between $p 16$ deletion and prognosis directly, $p 16$ deletion might be an important biological factor associated with the histological grade of MPNST that can be used to predict prognosis.

\section{Conclusion}

FISH for NF1 and $p 16$ deletions, which are observed frequently in high-grade MPNSTs, might be a useful ancillary diagnostic tool for differentiating MPNST from other mimicking spindle cell and pleomorphic sarcomas.

\footnotetext{
Abbreviations

FISH: Fluorescence in situ hybridization; H3K27me3: Histone 3 trimethylated on lysine 27; IHC: Immunohistochemistry; LMS: Leiomyosarcoma;

MFS: Myxofibrosarcoma; MPNST: Malignant peripheral nerve sheath tumor; NF1: Neurofibromatosis 1; NF1M: Neurofibromatosis 1-associated malignant peripheral nerve sheath tumor; NNF1M: Non-neurofibromatosis 1-associated malignant peripheral nerve sheath tumor; SS: Synovial sarcoma;

UPS: Undifferentiated pleomorphic sarcoma
}

\section{Acknowledgements}

The authors thank the following pathologists and clinicians for kindly contributing case materials and clinical follow-up information: Akihisa Saito, Institute for Clinical Research, National Hospital Organization Kure Medical Center and Chugoku Cancer Center, Hiroshima, Japan; Arafumi Maeshima, Department of Pathology, National Hospital Organization Tokyo Medical Center, Tokyo, Japan; Daisuke Noda, Department of Otolaryngology Head and Neck Surgery and Takashi Tsuchiya and Masato Sugawara, Department of Orthopedic Surgery, Yamagata University, Faculty of Medicine, Yamagata, Japan; Hajime Umezu, Division of Pathology, Niigata University Medical and Dental Hospital, Niigata, Japan; Hiroyuki Iwaki, Department of Pathology and Yasuharu Kunishima, Department of Urology, Sunagawa City Medical Center, Hokkaido, Japan; Hiroyuki Kawashima, Department of Orthopedic Surgery, Graduate School of Medical and Dental Sciences, Niigata University, Niigata, Japan; Johji Imura, Department of Diagnostic Pathology, Graduate School of Medicine and Pharmaceutical Sciences, University of Toyama, Toyama, Japan;
Katsushige Yamashiro, Division of Pathology, National Hospital Organization, Hokkaido Cancer Center, Hokkaido, Japan; Michiko Yamashita and Yoshiyuki Fujii, Division of Pathology, Tokushima Red Cross Hospital, Tokushima, Japan; Satoko Morohashi, Department of Pathology and Bioscience, Hirosaki University, Graduate School of Medicine, Aomori, Japan; Shigeri Ooba, Ooba Clinic, Hokkaido, Japan; Shingo Sakashita, Department of Pathology, Tsuchiura Kyodo General Hospital, Ibaraki, Japan; Takahiro Sugiyama, Division of Surgical Pathology, Chiba Cancer Center, Chiba, Japan; Takashi Kawasaki, Department of Pathology, Niigata Cancer Center Hospital, Niigata, Japan; and Yuji Harada, Department of Surgical Pathology, Shimane University Faculty of Medicine, Shimane, Japan.

\section{Authors' contributions}

SS participated in the design of the study, performed the pathological analysis, and drafted the manuscript. KS, KT, TS, MT, and HT helped with the pathological analysis. TA conducted the fluorescence in situ hybridization. TK and $T T$ performed the immunohistochemistry. ME examined the clinical data of the cases. TH conceived the study, participated in its design and coordination, and helped draft the manuscript. All authors read and approved the final manuscript.

\section{Funding}

No funding.

\section{Availability of data and materials \\ Not applicable.}

\section{Declarations}

Ethics approval and consent to participate

This research was approved by the IRB of Sapporo Medical University Hospital under permit number 272-107. The IRB approved an opt-out informed consent approach for a retrospective, non-interventional study.

\section{Consent for publication}

Not applicable.

\section{Competing interests}

The authors declare that they have no conflicts of interest.

\section{Author details}

'Department of Surgical Pathology, Sapporo Medical University, School of Medicine, South 1, West 16, Chuo-ku, Sapporo, Hokkaido 060-8543, Japan. ${ }^{2}$ Department of Orthopedic Surgery, Sapporo Medical University, School of Medicine, Sapporo, Hokkaido 060-8543, Japan.

Received: 12 January 2021 Accepted: 17 August 2021

Published online: 30 August 2021

\section{References}

1. Asano N, Yoshida A, Ichikawa H, et al. Immunohistochemistry for trimethylated $\mathrm{H} 3 \mathrm{~K} 27$ in the diagnosis of malignant peripheral nerve sheath tumours. Histopathology. 2017;70:385-93.

2. Cleven AH, Sannaa GA, Briaire-de Bruijn I, et al. Loss of H3K27 trimethylation is a diagnostic marker for malignant peripheral nerve sheath tumors and an indicator for an inferior survival. Mod Pathol. 2016;29:582-90.

3. Perry A, Roth KA, Banerjee R, Fuller CE, Gutmann DH. NF1 deletions in S-100 protein-positive and negative cells of sporadic and neurofibromatosis 1 (NF1)-associated plexiform neurofibromas and malignant peripheral nerve sheath tumors. Am J Pathol. 2001;159:57-61.

4. Suzuki K, Yasuda T, Hori T, Watanabe K, Kanamori M, Kimura T. An intraosseous malignant peripheral nerve sheath tumor of the lumbar spine without neurofibromatosis: Case report and review of the literature. Oncol Lett. 2014;7:1965-9.

5. Perry A, Kunz SN, Fuller CE, et al. Differential NF1, p16, and EGFR patterns by interphase cytogenetics (FISH) in malignant peripheral nerve sheath tumor (MPNST) and morphologically similar spindle cell neoplasms. J Neuropathol Exp Neurol. 2002;61:702-9.

6. Endo M, Kobayashi C, Setsu N, et al. Prognostic significance of p14ARF, p15INK4b, and p16INK4a inactivation in malignant peripheral nerve sheath tumors. Clin Cancer Res. 2011;17:3771-82. 
7. Miettinen MM, Antonescu CR, Fletcher CDM, et al. Histopathologic evaluation of atypical neurofibromatous tumors and their transformation into malignant peripheral nerve sheath tumor in patients with neurofibromatosis 1-a consensus overview. Hum Pathol. 2017:67:1-10.

8. Sugita $\mathrm{S}$, Asanuma $\mathrm{H}$, Hasegawa T. Diagnostic use of fluorescence in situ hybridization in expert review in a phase 2 study of trabectedin monotherapy in patients with advanced, translocation-related sarcoma. Diagn Pathol. 2016;11:37.

9. Le Guellec S, Macagno N, Velasco V, et al. Loss of H3K27 trimethylation is not suitable for distinguishing malignant peripheral nerve sheath tumor from melanoma: a study of 387 cases including mimicking lesions. Mod Pathol. 2017;30:1677-87.

10. Makise N, Sekimizu M, Kubo T, et al. Extraskeletal osteosarcoma: MDM2 and H3K27me3 analysis of 19 cases suggest disease heterogeneity. Histopathology. 2018;73:147-56.

11. Makise N, Sekimizu M, Konishi E, et al. H3K27me3 deficiency defines a subset of dedifferentiated chondrosarcomas with characteristic clinicopathological features. Mod Pathol. 2019;32:435-45

12. Marchione DM, Lisby A, Viaene AN, et al. Histone H3K27 dimethyl loss is highly specific for malignant peripheral nerve sheath tumor and distinguishes true PRC2 loss from isolated H3K27 trimethyl loss. Mod Pathol. 2019:32:1434-46.

13. Bui NQ, Przybyl J, Trabucco SE, Frampton G, Hastie T, van de Rijn M, et al. A clinico-genomic analysis of soft tissue sarcoma patients reveals CDKN2A deletion as a biomarker for poor prognosis. Clin Sarcoma Res. 2019;9:12.

14. Knösel T, Altendorf-Hofmann A, Lindner L. Loss of p16(INK4a) is associated with reduced patient survival in soft tissue tumours, and indicates a senescence barrier. J Clin Pathol. 2014;67:592-8.

15. Huang HY, Illei PB, Zhao Z, et al. Ewing sarcomas with p53 mutation or p16/ p14ARF homozygous deletion: a highly lethal subset associated with poor chemoresponse. J Clin Oncol. 2005;23:548-58.

\section{Publisher's Note}

Springer Nature remains neutral with regard to jurisdictional claims in published maps and institutional affiliations.

Ready to submit your research? Choose BMC and benefit from:

- fast, convenient online submission

- thorough peer review by experienced researchers in your field

- rapid publication on acceptance

- support for research data, including large and complex data types

- gold Open Access which fosters wider collaboration and increased citations

- maximum visibility for your research: over $100 \mathrm{M}$ website views per year

At $\mathrm{BMC}$, research is always in progress.

Learn more biomedcentral.com/submissions 